\title{
Modular Test Facility for HTS Insert Coils
}

\author{
V. Lombardo, A. Bartalesi, E. Barzi, M. Lamm, D. Turrioni and A.V. Zlobin
}

\begin{abstract}
The final beam cooling stages of a Muon Collider may require DC solenoid magnets with magnetic fields in the range of 40-50 $\mathrm{T}$. In this paper we will present a modular test facility developed for the purpose of investigating very high field levels with available 2G HTS superconducting materials. Performance of available conductors is presented, together with magnetic calculations and evaluation of Lorentz forces distribution on the HTS coils. Finally a test of a double pancake coil is presented.
\end{abstract}

Index Terms-High Field Solenoids, High Temperature Superconductors, Muon Collider, 2G HTS YBCO Conductor.

\section{INTRODUCTION}

I onization cooling for muon beams will require the development of a 6D Helical Cooling Channel [1, 2] and very high-field solenoids [3-5]. Both these configurations may make use of HTS materials to generate the required magnetic fields of operation. Second generation High Temperature Superconductors (2G HTS) show very high performances both in terms of $\mathbf{J}_{\mathrm{c}}$ at very high fields and of maximum allowable stress before any critical current degradation occurs. Thanks to its electrical and mechanical performances as well as its geometry, YBCO based conductors are considered for practical applications in both helical and straight high field solenoids for muon beams cooling.

The scope of the present work was to develop a facility to test multiple units of HTS double pancake coils with maximum flexibility in terms of conductor and coils geometry as well as winding techniques (i.e. single layer tape, ROEBLE cables...). The setup should allow for basic studies on small coils and allocate room for scale up within the $77 \mathrm{~mm}$ cold bore of a hybrid $\mathrm{Nb}_{3} \mathrm{Sn} / \mathrm{NbTi}$ magnet to perform in-field, low temperature studies. In the following, an overview of the chosen conductor for coil winding will be given, together with an overview of the key design aspects supported by magnetic

Manuscript received 20 October 2009. This work was supported in part by the U.S. Department of Energy.

V.Lombardo is with Fermi National Accelerator Laboratory, P.O. Box 500, Batavia, IL 60510, USA (e-mail: lombardo@fnal.gov).

A.Bartalesi is with Fermi National Accelerator Laboratory, P.O. Box 500, Batavia, IL 60510, USA (e-mail: antoniob@fnal.gov) and University of Pisa.

E. Barzi is with Fermi National Accelerator Laboratory, P.O. Box 500, Batavia, IL 60510, USA (e-mail: barzi@fnal.gov).

M. Lamm is with Fermi National Accelerator Laboratory, P.O. Box 500, Batavia, IL 60510, USA (e-mail: lamm@fnal.gov).

D. Turrioni is with Fermi National Accelerator Laboratory, P.O. Box 500, Batavia, IL 60510, USA (e-mail: turrioni@fnal.gov).

A. V. Zlobin is with Fermi National Accelerator Laboratory, P.O. Box 500, Batavia, IL 60510, USA (e-mail: zlobin@fnal.gov). and mechanical analysis of the limit scenario where all the available volume within the facility is occupied with HTS pancakes. Finally, the results of a double pancake coil test are presented as commissioning of the facility.

\section{SECOND GENERATION HTS CONDUCTOR}

\section{A. Conductor Details}

In the following some of the properties of the $2 \mathrm{G}$ HTS conductor chosen for the first coils winding are summarized. The cross-section of the tape consists of a $50 \mu \mathrm{m}$ Hastelloy substrate which provides overall mechanical support to the conductor, a buffer stack of about $0.2 \mu \mathrm{m}, 1 \mu \mathrm{m}$ YBCO layer and $2 \mu \mathrm{m}$ of silver. The tape is then finished with a total of 40 $\mu \mathrm{m}$ of copper stabilizer (one layer of $20 \mu \mathrm{m}$ per side). Available conductor shows critical current values in nitrogen under self field condition ranging from $90 \mathrm{~A}$ to $120 \mathrm{~A}$.

TABLE I. 2G HTS CONDUCTOR PROPERTIES

\begin{tabular}{cc}
\hline \hline Tape Manufacturer & Superpower \\
2G Tape & SCS4050 \\
Nominal Width & $4 \mathrm{~mm}$ \\
Nominal Thickness & $0.1 \mathrm{~mm}$ \\
Stabilizer & Copper $(2 \times 20 \mu \mathrm{m})$ \\
Substrate & Hastelloy® $\mathrm{C} 276(50 \mu \mathrm{m})$ \\
YBCO layer thickness & $1 \mu \mathrm{m}$ \\
\hline \hline
\end{tabular}

\section{B. Conductor Characterization}

Second generation HTS YBCO tapes show strong anisotropic behavior with respect to field orientation. For this reason, an accurate understanding of how critical current scales with field and field orientation is needed in order to precisely include this effect into solenoids design. Several short samples have been tested at Fermilab on a rotating sample holder at different angle orientations up to a magnetic field of $15 \mathrm{~T}$. Tests have been run within an anti-cryostat equipped with a heater and a needle valve. Continuously regulating the flux of helium within the anti-cryostat and the heater power, the sample temperature could be held steady for the whole duration of a test run. Using this technique, critical current dependency on operating sample temperature has been evaluated in a range from $4.2 \mathrm{~K}$ up to $33 \mathrm{~K}$. In Fig 1 through 4 some results have been summarized. Critical current has been defined using a $1 \mu \mathrm{V} / \mathrm{cm}$ criterion for all the following plots. 


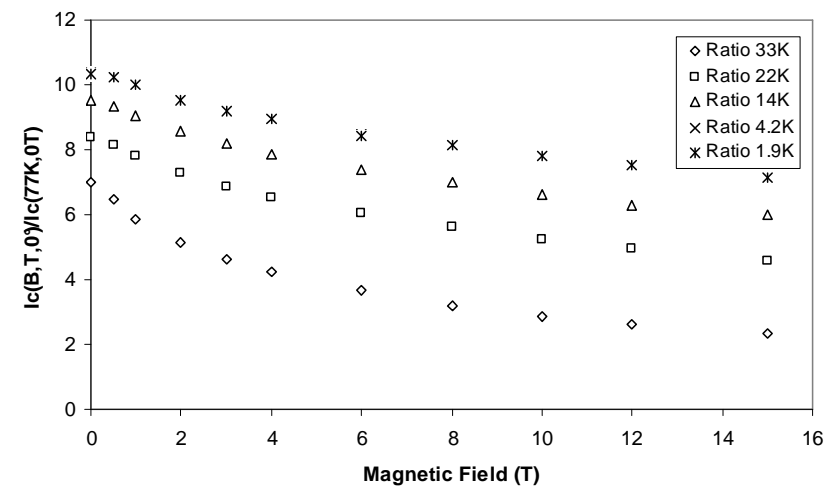

Fig. 1. $\mathrm{I}_{\mathrm{c}}$ ratio measured with magnetic field at $0^{\circ}$ with respect to a-b plane.

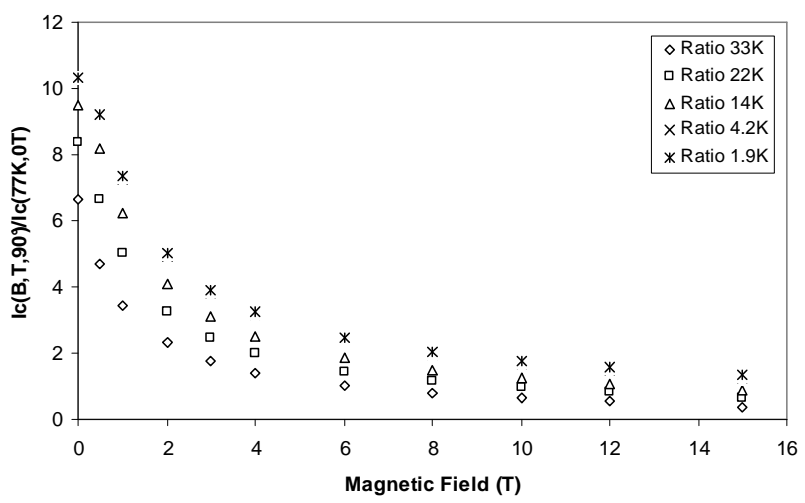

Fig. 2. $\mathrm{I}_{\mathrm{c}}$ ratio measured with magnetic field at $90^{\circ}$ with respect to a-b plane.

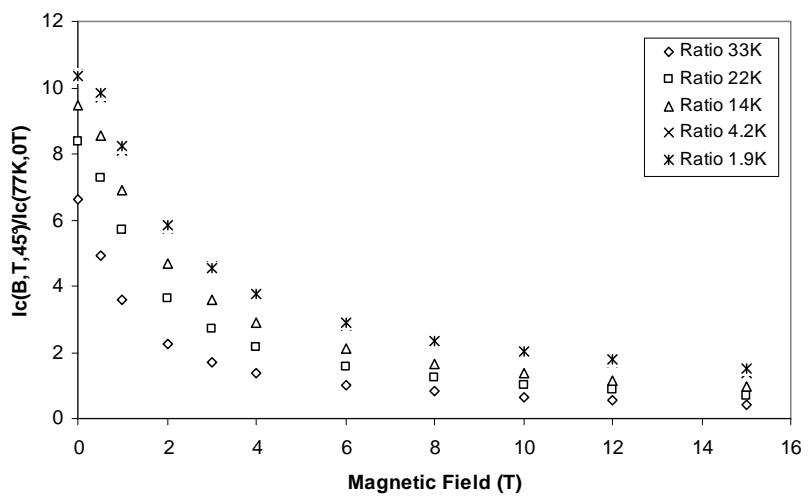

Fig. 3. $\mathrm{I}_{\mathrm{c}}$ ratio measured with magnetic field at $45^{\circ}$ with respect to a-b plane.

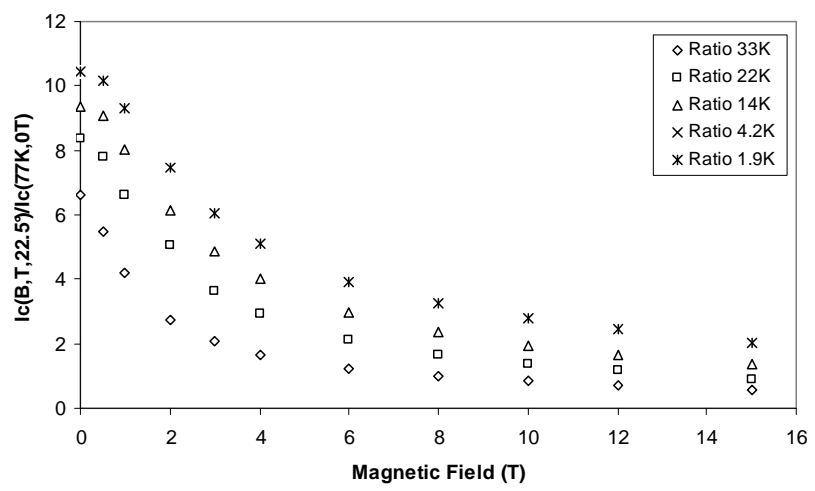

Fig. 4. $I_{c}$ ratio measured with magnetic field at $22.5^{\circ}$ with respect to a-b plane.

Despite the high performance of the conductor in parallel field, very sharp drops in critical current are noticeable even for small angles. Anisotropy ratio, defined as the ratio between critical current under parallel field and perpendicular field, show value as high as 7 around $15 \mathrm{~T}$ (Fig. 5). It is interesting to notice that no major improvement is noticeable when the conductor is operated in superfluid helium $(1.9 \mathrm{~K})$.

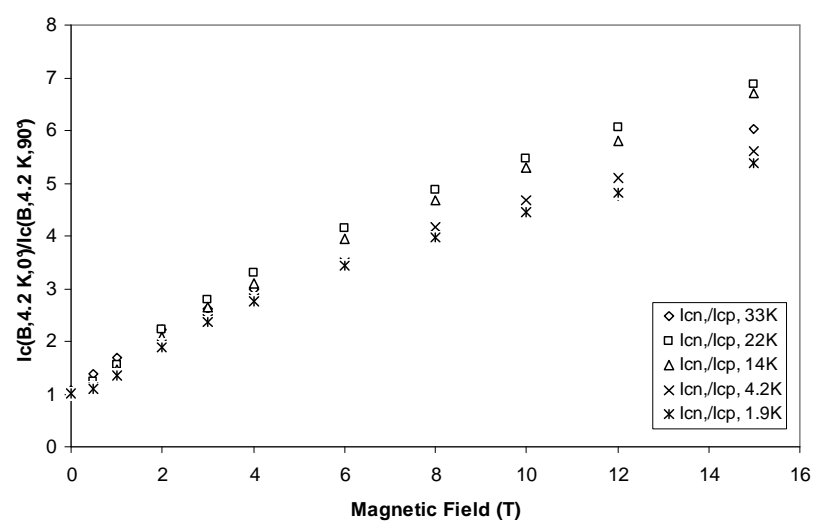

Fig. 5. Anisotropy ratio of $2 \mathrm{G}$ HTS tape as a function of temperature and field

\section{MOdUlar TeSt FACILITY FOR HTS INSERT COILS}

The modular facility has been designed to accommodate up to 14 double pancake units wound out of SCS4050 YBCO tape (see Table II), or any other number of pancakes made using different conductors having the same overall volume. The facility features an internal stainless steel support for double pancake coils stacking and copper current leads designed as relatively thin circular sectors for minimal room occupancy (see Fig. 6). Current lead sections have been optimized to carry up to $2 \mathrm{kA}$ by minimizing helium evaporation due to conduction and Joule heating. Each double pancake unit is wound onto a G-10 support featuring a groove to simplify the winding procedure and prevent conductor movements during test that might otherwise cause quench. Following this approach, different conductors and coil geometries can be accommodated into the structure by simply redesigning small, rather inexpensive, G-10 supports. The minimum internal diameter has been chosen in accordance with bending limitations of the conductor [6] and to allow enough room for the insertion of a Hall probe to perform measurement of the axial field produced by the inserts. The maximum outer diameter has instead been fixed because of clearance limitations due to the $77 \mathrm{~mm}$ cold bore of the outer $\mathrm{Nb}_{3} \mathrm{Sn} / \mathrm{NbTi}$ magnet.

TABLE II. GEOMETRY OF LARGEST POSSIBLE CONFIGURATION

$\begin{array}{cc}\text { Coil inner diameter } & 19 \mathrm{~mm} \\ \text { Coil outer diameter } & 62 \mathrm{~mm} \\ \text { Maximum number of coils } & \text { 14 SCS4050 double pancakes }\end{array}$

The two shell current leads are connected to copper rings that can be moved as far apart from each other as needed by the coil geometry to be built. Room not needed for superconducting coils can be easily filled using G-10 dummies, as shown in Fig. 6. 


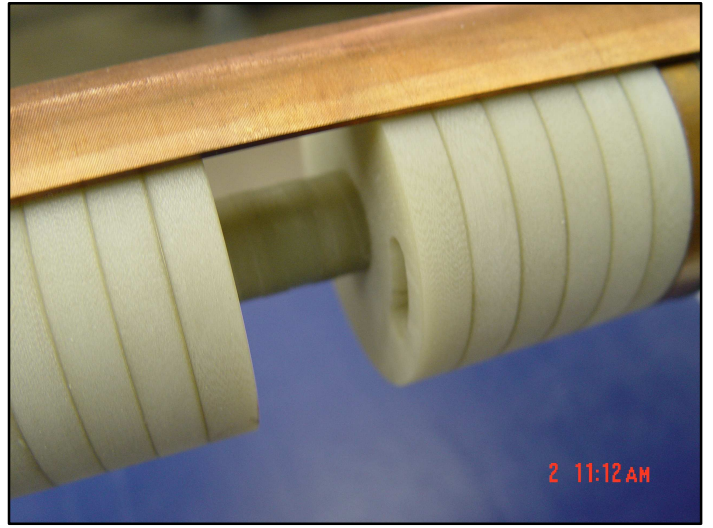

Fig. 6. Details of copper current leads, dummy coils and coil G-10 supports.

\section{A. Magnetic Analysis}

In order to study the capabilities of the facility and finalize the mechanical design, several simulations have been run focusing on the largest configuration possible, which corresponds to having all the available volume filled with conductor. In this case, the magnetic field distribution generated by the HTS insert in a $14 \mathrm{~T}$ background field would be the one shown in Fig. 7. This configuration corresponds to 14 SCS4050 double pancakes with the geometry stated in Table II.

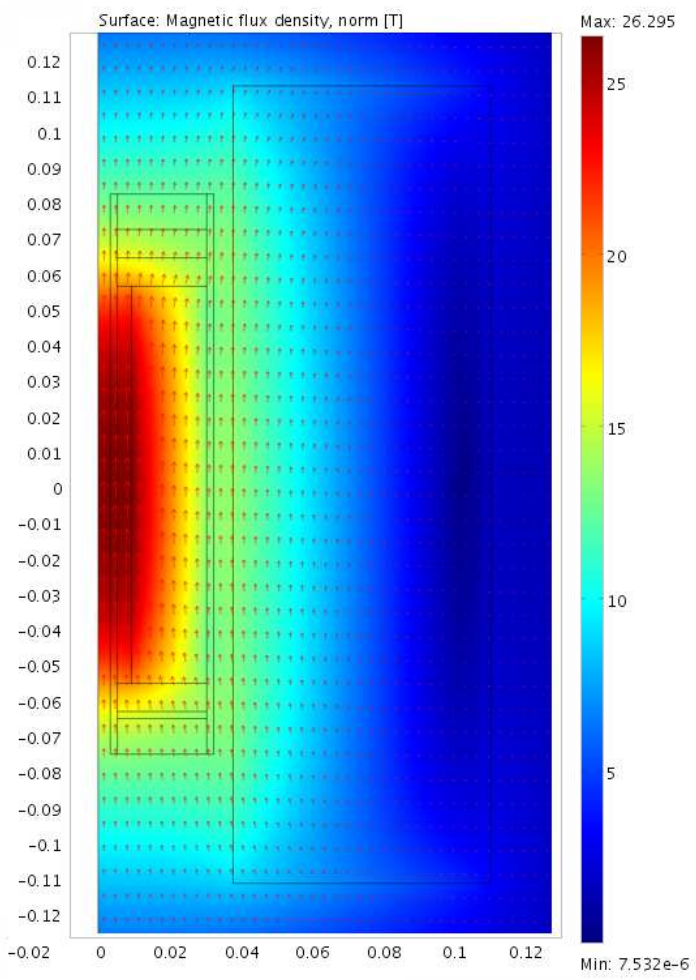

Fig. 7. Magnetic Field distribution due to external $\mathrm{NbTi} / \mathrm{Nb}_{3} \mathrm{Sn}$ magnet and HTS Insert coil $\left(I_{c}=294 A\right)$

To evaluate the magnetic field distribution in Fig. 7, the $I_{c}$ of the coil needs to be evaluated. In order to perform this calculation, critical current data at $4.2 \mathrm{~K}$ from Fig. 1 to 4 . have been fitted using the analytical expression in [6]. The distribution of the magnetic field on the HTS volume can be easily evaluated using commercial FEM packages (Fig. 8). Using this piece of information and an analytical expression for $I_{c}(B, \theta)$, a mapping of the critical current within the whole coil can be evaluated (Fig. 8). Iterating this process, one can find that the critical current is - as expected - limited at the edges of the solenoid, due to the angle and the intensity at which the magnetic field impact on the solenoid itself. As one can see from Fig. 8, angles are limited to $\pm 15.4^{\circ}$ for this particular geometry, due to the fact that the coil is used as an insert within an external (bigger) magnet. According to this calculation, the estimated critical current is equal to $\sim 294 \mathrm{~A}$ (Fig. 8).
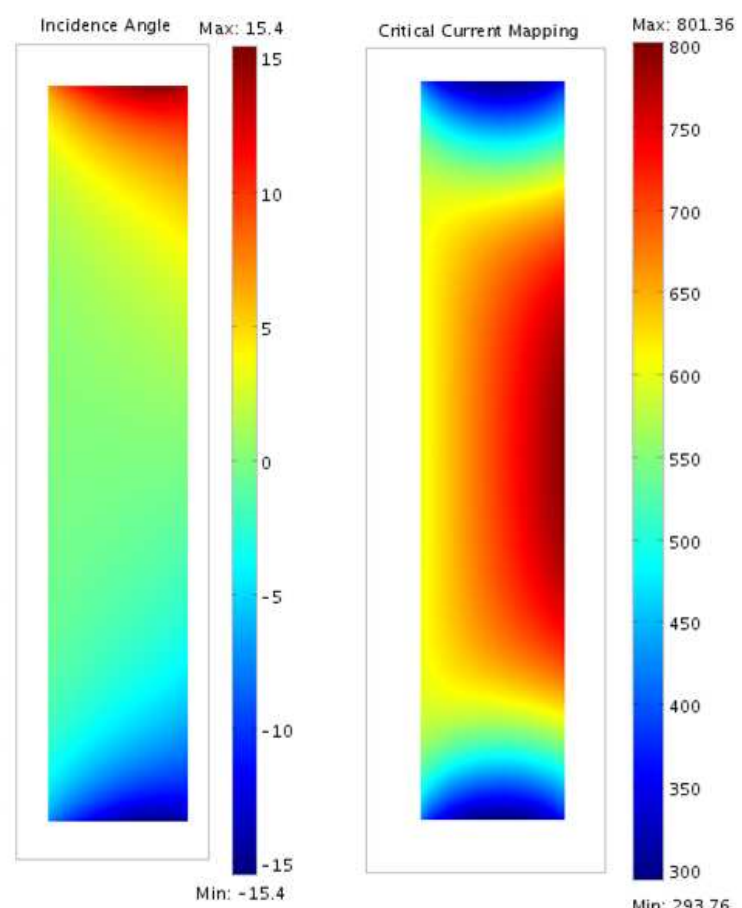

Fig. 8. Incidence angle of magnetic field on the HTS coil (left) Critical current mapping on the HTS coil. (right)

\section{B. Mechanical Analysis}

A non-linear finite element model has been made to evaluate the stresses in the HTS coil supported by an outer stainless steel support structure and inserted in a background field of $14 \mathrm{~T}$. Both thermal contractions due to cooling to liquid helium and Lorentz forces have been taken into account in modeling the coil using contact elements between surfaces.

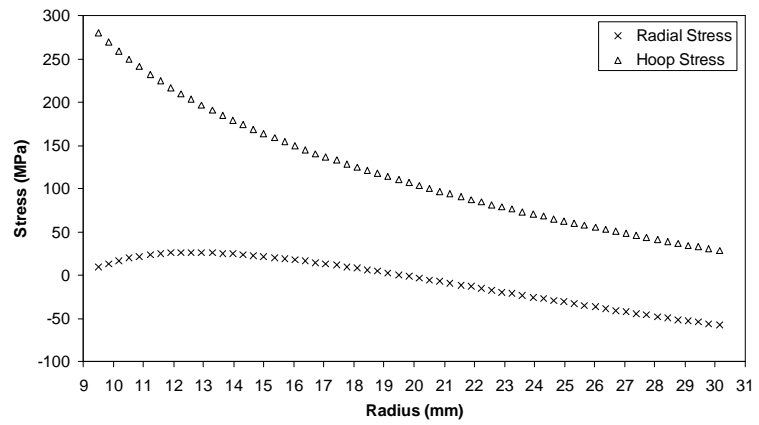

Fig. 9. Azimuthal and Radial stress on the $2 \mathrm{G}$ HTS coil at I=294A. 
In Fig 9, radial and azimuthal stresses are plotted along the radius of the coil. In this calculation, a maximum operational current of $294 \mathrm{~A}$, as evaluated in the previous paragraph, and no mechanical pre-stress were used, other than the one provided by the cool down to liquid helium. The calculated maximum azimuthal stress is below $300 \mathrm{MPa}$, which is less than half what the conductor can bear before showing any degradation in critical current [7].

\section{COMMISSIONING OF INSERT TEST FACILITY (ITF)}

Following the complete assembly and instrumentation of the probe, a double pancake unit has been wound, instrumented and tested. In Fig. 10 the bottom part of the final assembly of the probe is shown. The double pancake unit has been wound out of the SCS4050 tape characterized in Section II, using a co-wound 1.2 mil turn to turn adhesive Kapton tape as electrical insulation. The coil has been equipped with a pair of voltage taps to monitor quench development and with a Cernox sensor to detect any increase in temperature during operation. The two coils are separated by a thin G-10 shim. The conductor has been soldered using In52-Sn48 to the two copper rings using a wider $(12 \mathrm{~mm})$ tape as a bridge to minimize Joule heating during operation. The last layer of the coil has been brushed with Stycast 2850. After curing of the Stycast, the coil has been cooled down to $77 \mathrm{~K}$ and excited at a ramp rate of $2 \mathrm{~A} / \mathrm{s}$. Fig. 11 shows the VI curve acquired during the first ramp of the coil. The coil remained in a superconducting state up to $\sim 70 \mathrm{~A}$. The transition to the normal state was very sharp, and the $30 \%$ reduction in critical current (from the nominal $100 \mathrm{~A}$ ) was consistent with the reduction expected from the perpendicular component of the self field on the innermost winding.

The coil was subsequently inserted in the outer solenoid, cooled with liquid Helium and excited in a background field of $14 \mathrm{~T}$ at a ramp rate of. $3 \mathrm{~A} / \mathrm{s}$. The insert coil remained in a superconducting state up to $523 \mathrm{~A}$ when the protection system tripped and the stored energy released.

TABLE III. PARAMETERS OF HTS TEST COIL

\begin{tabular}{cc} 
TABLE III. PARAMETERS OF HTS TEST COIL \\
\hline \hline Coil inner diameter & $60 \mathrm{~mm}$ \\
Coil outer diameter & $62 \mathrm{~mm}$ \\
Conductor & Superpower SCS4050 \\
Winding geometry & Double Pancake \\
Joints to copper & Superpower SCS12050 \\
Insulation & 1.2 mil turn to turn Kapton \\
\hline \hline
\end{tabular}

\section{CONCLUSION}

In this work a modular test facility developed to investigate the magnetic field levels achievable with available 2G HTS superconducting materials has been described. The critical current of YBCO short samples measured at Fermilab has been presented and used to estimate the performance of the largest coil that can be wound and tested in the Insert Test Facility (ITF). For this limit configuration, magnetic field distribution and stresses on the coils have been evaluated and discussed. Finally a double pancake coil test has been presented as part of the ITF commissioning. Such commissioning will include testing of additional coil units.

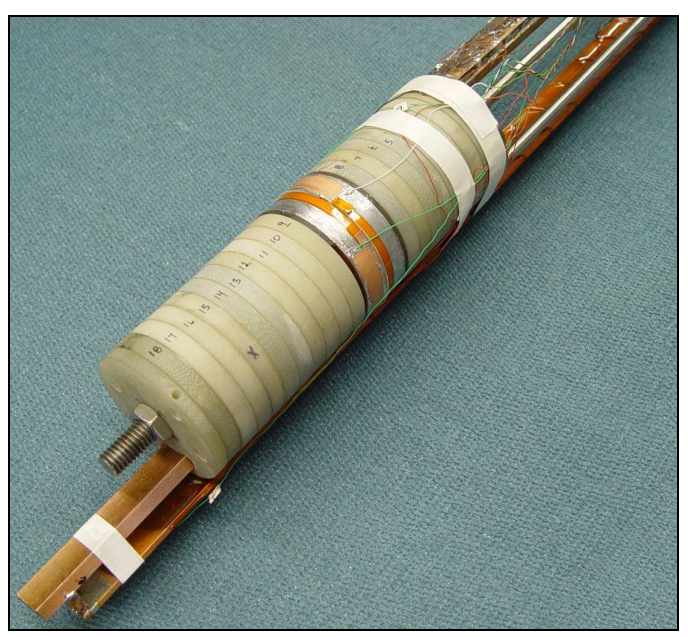

Fig. 10 - Detail of the bottom part of the probe with one mounted and instrumented double pancake coil.

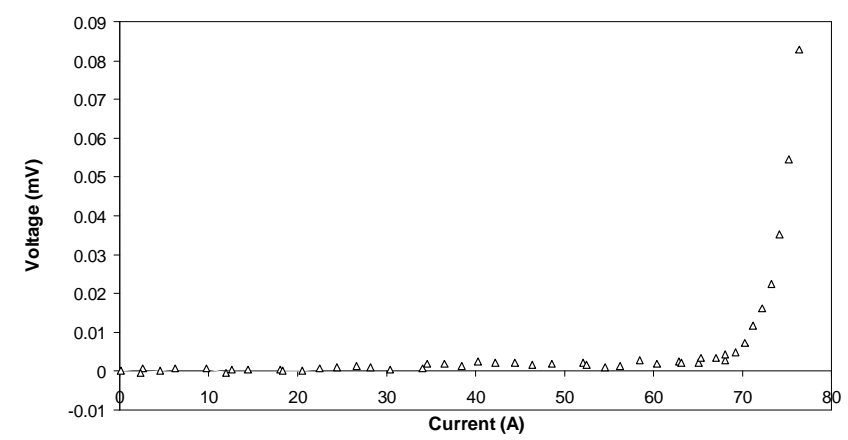

Fig. 11 - VI curve for one double pancake unit tested in nitrogen under self field conditions.

\section{ACKNOWLEDGMENT}

The authors thank the special people of our Superconductor R\&D group at Fermilab, Al Rusy, Tom Van Raes and Sean Johnson whose skills and commitment were essential for this work.

\section{REFERENCES}

[1] S. A. Kahn et al., "Magnet Systems for Helical Muon Cooling Channels", Proceedings of PAC07, Albuquerque, NM, June 2007.

[2] V. V. Kashikhin et al., "Design Studies of Magnet Systems For Muon Helical Cooling Channels", EPAC2008, Genoa, Italy, June 2008.

[3] S. A. Kahn et al., "High Field Solenoid Magnets for Muon Cooling", Proceedings of EPAC2006, Edinburgh, Scotland, pp. 2634-2536.

[4] S. A. Kahn et al., "A High Field HTS Solenoid for Muon Cooling", Proceedings of PAC07, Albuquerque, NM, June 2007.

[5] V. V. Kashikhin et al., "Study of High Field Superconducting Solenoids for Muon Beam Cooling", IEEE Trans. Appl. Sup., V. 18, No. 2, pp. 928-932 (2007).

[6] V.Lombardo et al., "Study of HTS Insert Coils for High Field Solenoids", accepted for publication in Advances in Cryogenic Engineering, AIP.

[7] Y. Xie et al, "Status of HTS Projects at SuperPower: 2G HTS Wire and Cable. Workshop on Superconducting DC Transmission and Distribution", Feb. 25, 2009. 